\title{
Impact of Thrombosis and Bleeding in Patients with Severe COVID-19 versus Other Viral Pneumonias in the Context of Extracorporeal Membrane Oxygenation
}

\author{
Anna Weatherill, MBChB ${ }^{1}$ Mike Laffan, DM ${ }^{1,2}$ Mihaela Gasper, MSc ${ }^{3}$ Paolo Bianchi, MD ${ }^{4,5,6}$ \\ Maurizio Passariello, EDIC ${ }^{4}$ Suveer Singh, $\mathrm{PhD}^{4,5}$ James Doyle, EDICM ${ }^{4}$ Brijesh Patel, $\mathrm{PhD}^{4,5}$ \\ Stephane Ledot, $\mathrm{MD}^{4,5,6}$ Benjamin Garfield, $\mathrm{PhD}^{4,5}$ Deepa J. Arachchillage, MD ${ }^{1,2,3}$ \\ ${ }^{1}$ Department of Haematology, Imperial College Healthcare NHS Trust \\ Imperial College London, London, United Kingdom \\ ${ }^{2}$ Centre for Haematology, Imperial College London, London, United \\ Kingdom \\ ${ }^{3}$ Department of Haematology, Royal Brompton \& Harefield Hospitals, \\ part of Guy's and St Thomas's NHS Foundation Trust, London, United \\ Kingdom \\ ${ }^{4}$ Department of Adult Intensive Care, Royal Brompton \& Harefield \\ Hospitals, part of Guy's and St Thomas's NHS Foundation Trust, \\ London, United Kingdom \\ Semin Thromb Hemost 2022;48:118-123. \\ Address for correspondence Deepa J. Arachchillage, MD, \\ Department of Haematology, Imperial College Healthcare NHS Trust \\ and Imperial College London, Hammersmith Hospital, 4th Floor, \\ Commonwealth Building, Du Cane Road, London W12 ONN, United \\ Kingdom (e-mail: d.arachchillage@imperial.ac.uk). \\ ${ }^{5}$ Division of Anesthetics, Pain Medicine, and Intensive Care, \\ Department of Surgery and Cancer, Imperial College London, \\ London, United Kingdom \\ ${ }^{6}$ Department of Anesthesia, Royal Brompton \& Harefield Hospitals, \\ part of Guy's and St Thomas's NHS Foundation Trust, London, United \\ Kingdom
}

Extracorporeal membrane oxygenation (ECMO) could be life saving for patients with severe acute respiratory syndrome coronavirus 2 (SARS-CoV-2)-induced severe respiratory failure not responding to optimal conventional management, including mechanical ventilation and proning. ${ }^{1}$ However, thrombosis and/or bleeding may be major complications of patients supported with ECMO.2

The aim of this single-center retrospective observational study in a UK ECMO center was to assess whether thrombotic or bleeding phenotypes differ in patients with severe coronavirus disease 2019 (COVID-19) pneumonia treated with veno-venous (VV)-ECMO compared to those who received VV-ECMO for other viral pneumonias. Mortality rate up to 120 days after VV-ECMO initiation was also assessed, as well as the factors directly contributing to death.

Fifty-four consecutive COVID-19 patients supported with VVECMO from March $17^{\text {th }}, 2020$ to May $26^{\text {th }}, 2020$ were compared with an age and sex-comparable cohort of 43 patients with nonCOVID-19 viral pneumonia from April 5th 2018 to January $19^{\text {th }}$, 2020. Of the 43 non-COVID-19 patients, the majority of them had Influenza A (38/43, 88.4\%), 4.6\% (2/43) had non-coronavirus disease 2019 (non-COVID-19), one patient had co-infection of parainfluenza and rhinovirus and the remaining two patients had human metapneumovirus and adenovirus, respectively. All patients had a confirmed diagnosis of viral infection from polymerase chain reaction nasopharyngeal swabs. Both cohorts were managed using the same scanning and anticoagulant protocols. ${ }^{3}$ The study was approved by the Research Ethics Committee and the local Research and Development Office (reference number: 20/EE/0160).

Anticoagulation with unfractionated heparin (UFH) was managed as described in our previous study. ${ }^{3}$ Argatroban was used in patients with heparin-induced thrombocytopenia (HIT) or when heparin resistance was evident. ${ }^{4}$

All thrombotic and bleeding complications were identified on routine or clinically indicated $\mathrm{CT}$ or ultrasound (US) imaging. Routine whole-body CT imaging was performed in all patients within 24 hours of admission for VV-ECMO. Routine upper and lower limb compression US and/or CT venography was performed within 24 hours of ECMO decannulation. Additional imaging was performed as indicated for clinical suspicion of thrombosis or bleeding.

Bleeding events were defined as major or clinically relevant minor hemorrhages according to ISTH classification. ${ }^{5,6}$ Diagnosis of HIT was performed as described previously ${ }^{3}$ (appendix page 1). Anticoagulant levels and target on the day of thrombotic or bleeding events were recorded. published online August 13, 2021
Issue Theme Maintaining Hemostasis and Preventing Thrombosis in COVID-19-Part III; Guest Editors: Emmanuel J. Favaloro, PhD, FFSc (RCPA) and Giuseppi Lippi, MD (c) 2021. Thieme. All rights reserved. Thieme Medical Publishers, Inc., 333 Seventh Avenue, 18th Floor, New York, NY 10001, USA
DOI https://doi.org/ 10.1055/s-0041-1732371. ISSN 0094-6176. 
Results are presented as percentages for categorical data, median and range for skewed continuous data, and means (95\% confidence interval). Groups were compared using the Chi-square test for categorical data, and the $t$-test for continuous data (as appropriate). $p$-Values $<0.05$ were considered statistically significant. Changes in blood results over time are presented in graphical format.

Baseline characteristics of the COVID-19 and non-COVID19 cohorts were comparable by age, sex, body-mass index (BMI), and co-morbidities (- Table 1). The duration of VVECMO was greater in patients with COVID-19 compared to non-COVID-19: median (range) was 19 (3-57) days versus 15 (3-60) days $(p=0.02)$. Laboratory parameters (averaged in the first 24 hours after initiation of ECMO) are presented in -Table 1. Platelet and neutrophil counts were higher in patients with COVID-19 compared to non-COVID-19. Median fibrinogen levels on admission were increased in both groups but were significantly higher in the COVID-19 cohort. There were no other laboratory parameter differences between groups in the 24 hours following initiation of ECMO.

In the COVID-19 cohort, there were 69 thrombotic events in 43/54 (80\%) patients: 39 (57\%) pulmonary emboli (PE), 19 (28\%) DVTs, and 11 (16\%) arterial thrombotic events (-Supplementary Table S1, available in the online version only). PE occurred in 36/54 (67\%) COVID-19 patients, DVT in $19 / 54$ (35\%), and arterial thrombosis in 10/54 (19\%). Twentytwo (41\%) COVID-19 patients had two or more events. Of the 69 thrombotic events, 36 events were diagnosed during or after VV-ECMO and 32/36 (89\%) of those events occurred while patients were receiving thromboprophylaxis or treatment dose anticoagulation.

PE was much more likely to occur in isolation 23/39 than with DVT 9/39 in the COVID-19 cohort. Of the DVTs in the COVID-19 cohort, 8/19 (42\%) were line associated. Of the 11 arterial thromboses in COVID-19 patients, 6/11 (55\%) were ischemic strokes and others were bowel ischemia (2/11), acute coronary syndrome (2/11), and renal infarction (1/11).

The timing of thrombotic events in relation to VV-ECMO is shown in - Supplementary Table $\mathbf{S 1}$ (available in the online version only): $33 / 69$ (48\%) thrombotic events (23 PE, four DVT, and six arterial thrombosis) occurred before VV-ECMO (identified either before or at the initiation of VV-ECMO) and 30/69 (43\%) events (16 PE, 11 DVT, and three arterial thrombosis) were diagnosed during VV-ECMO. Among those 30 thrombotic events that occurred during VV-ECMO, the median time on ECMO before the event was 9 days. Only 6/69 (9\%) events (four DVT and two arterial thrombosis) were diagnosed after VV-ECMO.

In the non-COVID-19 cohort, there were 34 thrombotic events in 26/43 patients (60\%): 11 (33\%) were PEs, 17 (52\%) DVTs, and six (18\%) arterial thrombosis. PE occurred in 11/43 (26\%) non-COVID-19 patients, DVT in 15/43 (35\%), and arterial thrombosis in 5/43 (12\%). Only six (14\%) non-COVID-19 patients had two or more events. 15/20 (75\%) events following the initiation of ECMO occurred while patients were receiving thromboprophylaxis or therapeutic anticoagulation.

As in COVID-19, PE in the non-COVID-19 cohort was more frequently diagnosed in isolation (10/11) than along- side DVT and a similar proportion of DVTs (41\%) was line associated. The anatomical distribution of arterial thrombosis was similar in the COVID-19 and non-COVID-19 cohorts. Of the five non-COVID-19 patients who developed arterial thrombosis, three patients developed ischemic stroke and the other two patients each developed bowel, splenic, and renal infarcts.

Thrombotic events in non-COVID-19 patients were distributed more evenly throughout the course of ECMO (prior, during and post-decannulation from ECMO) than for COVID-19 patients, in whom the majority of thromboses developed prior to or early in the course of ECMO ( - Supplementary Table S1, available in the online version only). Thirteen of the 34 (38\%) thrombotic events (nine PE, one DVT, three arterial thrombosis) occurred in 12 (27.9\%) non-COVID-19 patients before VV-ECMO. 10/34 (29\%) events (one PE, eight DVT, one arterial thrombosis) occurred in 10 (23.3\%) patients during VV-ECMO. Among the 10 thrombotic events that occurred during VV-ECMO, median time on ECMO before thrombotic event was 15 days. 11/34 (32\%) events (one PE, eight DVT, two arterial thrombosis) occurred in nine (21\%) patients after VV-ECMO.

Of the 19 and 17 patients from the COVID-19 and nonCOVID-19 cohorts who developed DVTs, 8/19 (42\%) and 7/17 (41\%) were central venous line associated.

There were 24 major bleeding events in 19 COVID-19 patients and $54 \%$ (13/24) of them were intracranial hemorrhages (ICHs) (- Supplementary Table S2, available in the online version only). Three of 24 major bleeding events were diagnosed on the day of initiation of VV-ECMO in patients who were not previously receiving treatment dose anticoagulation (all three of these were intracranial bleeds). The remaining 21 events occurred during VV-ECMO; all were preceded by thrombotic events and 15/21 occurred while patients were receiving UFH (11/20) or argatroban (4/20) at treatment doses. Median time on VV-ECMO before major bleeding event was 10 days (range 0-34 days). Clinically relevant minor bleedings were also more frequent: 25 events in $19 / 54$ patients.

There were 11 major bleeding events in nine non-COVID patients and 45\% (5/11) were ICH (-Supplementary Table S2, available in the online version only). Four of the 11 were diagnosed on the day of initiation of VV-ECMO (two intracranial and two gastrointestinal) (-Supplementary Table S2, available in the online version only). None of the four patients in whom these events occurred were receiving treatment dose anticoagulation before VV-ECMO.

Only three of these 11 cases of major bleeding were preceded by a thrombotic event. Only one major bleed occurred during administration of treatment dose anticoagulation (UFH with target anti-Xa level 0.3-0.5). The remaining events occurred off anticoagulation (5/11) or during administration of prophylactic UFH (target anti-Xa level 0.2-0.3)(5/11). Median time on VV-ECMO before major bleeding event was 2 days (range 0-18 days). Eighteen clinically significant minor bleedings occurred in 15/43 patients.

There was no clear difference in aPTT or PT between the patients who developed major bleeding and those who did 
Table 1 Baseline characteristics at the initiation of ECMO in patients with COVID-19 versus non-COVID-19 viral pneumonia

\begin{tabular}{|c|c|c|c|c|}
\hline Variable & Reference range & COVID-19 $(n=54)$ & Non-COVID-19 $(n=43)$ & $p$-Value \\
\hline Age, mean (SD) & - & $46.3(10.8)$ & $46.4(10.5)$ & 0.96 \\
\hline Gender, male & - & $40(74.1 \%)$ & $31(72.1 \%)$ & 1.0 \\
\hline BMI, mean (SD) & - & $29.8(6.4)$ & $30.4(7.2)$ & 0.65 \\
\hline Diabetes & - & $11(20.4 \%)$ & $11(25.6 \%)$ & 0.61 \\
\hline Ischemic heart disease & - & 0 & 0 & - \\
\hline Hypertension & - & $13(24.1 \%)$ & $9(20.9 \%)$ & 0.80 \\
\hline Previous VTE & - & 0 & $2(4.6 \%)$ & 0.50 \\
\hline Anticoagulant therapy & - & $0 / 15$ & $2(4.6 \%)$ & 1.00 \\
\hline Antiplatelet & - & $12(21.8 \%)$ & $7(16.3 \%)$ & 0.21 \\
\hline Haemoglobin g/L, median (range) & $130-160$ & $100.0(73.0-149.0)$ & $96.3(70.0-135.8)$ & 0.92 \\
\hline Platelets $\times 10^{9} / \mathrm{L}$, median (range) & $150-400$ & $254(69-545)$ & $130(46-454)$ & 0.0002 \\
\hline $\begin{array}{l}\text { White blood cells } \times 10^{9} / \mathrm{L} \text {, median } \\
\text { (range) }\end{array}$ & $4.1-11.1$ & $11.50(4.60-28.70)$ & $10.96(1.90-34.3)$ & 0.16 \\
\hline Neutrophils $\times 10^{9} / \mathrm{L}$, median (range) & $2.1-6.7$ & $9.60(3.8-26.40)$ & $7.41(1.40-21.45)$ & 0.04 \\
\hline $\begin{array}{l}\text { Lymphocytes } \times 10^{9} / \mathrm{L} \text {, median } \\
\text { (range) }\end{array}$ & $1.3-3.7$ & $0.80(0.30-5.10)$ & $0.72(0.10-2.5)$ & 0.19 \\
\hline PT seconds, median (range) & $10.2-13.2$ & $14.2(13.1-15.6)$ & $13.1(12.1-14.9)$ & 0.24 \\
\hline aPTT seconds, median (range) & $26.0-36.0$ & $55.3(35.1-201.2)$ & $45.4(33.7-177.4)$ & 0.97 \\
\hline $\begin{array}{l}\text { D-dimer } \mathrm{ng} / \mathrm{mL} \text { (D-dimer units), } \\
\text { median (range) }\end{array}$ & $0-500$ & $2,987(1,316-17,597)$ & $2,379(845-20,605)$ & 0.13 \\
\hline Fibrinogen $\mathrm{g} / \mathrm{L}$, median (range) & $1.5-4.5$ & $6.1(1.40-10.7)$ & $4.6(1.16-10.0)$ & 0.01 \\
\hline Antithrombin IU/dL, median (range) & $70-140$ & $65.0(20.0-137.0)$ & $65.5(14.0-138.0)$ & 0.45 \\
\hline ALT IU/L, median (range) & $8-40$ & $45.0(10.0-195.0)$ & $57.0(15.3-826)$ & 0.09 \\
\hline Bilirubin $\mu \mathrm{mol} / \mathrm{L}$, median (range) & $1-17$ & $14.5(5.0-108.0)$ & $19.7(5.0-64.0)$ & 0.08 \\
\hline $\begin{array}{l}\text { Creatinine clearance } \mathrm{mL} / \mathrm{min} \text {, median } \\
\text { (range) }\end{array}$ & & $88(17-182)$ & $66(23-172)$ & 0.08 \\
\hline CRP mg/L, median (range) & $0-10$ & $240.0(16.0-553.0)$ & $187.0(45.0-429.0)$ & 0.22 \\
\hline Ferritin $\mu \mathrm{g} / \mathrm{L}$, median (range) & $20-186$ & $889.0(68.0-14,863)$ & $910(80-15,620)$ & 0.8 \\
\hline LDH IU/mL, median (range) & $266-500$ & $1,084.5(570-3,293)$ & $1,067.1(510-3,880)$ & 0.7 \\
\hline Admission Murray score, mean (SD) & - & $3.43(0.29)$ & $3.10(0.43)$ & 0.002 \\
\hline Admission SOFA score, mean (SD) & - & $8.98(2.54)$ & $10.11(2.87)$ & 0.010 \\
\hline
\end{tabular}

Abbreviations: ALT, alanine transferase; aPTT, activated partial thromboplastin time; BMI, body mass index; COVID-19, coronavirus disease-19; CRP, C-reactive protein; ECMO, extracorporeal membrane oxygenation; LDH, lactate dehydrogenase; PT, prothrombin time; SD, standard deviation; SOFA, sequential organ failure assessment; VTE, venous thromboembolism.

Note: Bold texts and values in the table indicate the differences between the two groups are significant.

not, in either cohort. Heparin anti-Xa levels were generally higher in the COVID-19 patients who developed major hemorrhage than in those who did not (-Figs. 1 and $\mathbf{2}$ ). In general, anti-Xa levels were higher in the COVID-19 cohort throughout the course of ECMO because a greater proportion had confirmed thrombosis necessitating therapeutic dose anticoagulation (-Fig. 1D).

Murray score was higher in the COVID-19 group: mean 3.43 compared to 3.10 in the non-COVID-19 cohort $(p=0.002)$. Fewer COVID-19 patients required renal replacement therapy (RRT): $23 / 54$ (43\%) compared to $30 / 43$ (70\%), $p=0.008$. The proportion of COVID-19 patients who devel- oped multiorgan failure during ECMO (16/54, 30\%) was less than half that in non-COVID-19 (29/43, 67\%), $p=0.0002$.

Although 120-day-mortality was numerically higher in the non-COVID-19 cohort (12/43 [28\%]) compared to 9/54(17\%) in the COVID-19 cohort, this was not statistically significant $(p=0.21)$. Major bleeding was contributory to mortality in the majority of COVID-19 fatalities (5/9,55\%) compared to none in the non-COVID-19 group. - Supplementary Tables $\mathbf{5 3}$ and $\mathbf{S 4}$ (available in the online version only) show clinical details of the patients who died in each cohort.

To summarize, thrombosis and major bleeding are frequent complications in both COVID-19 and non-COVID-19 patients 
A

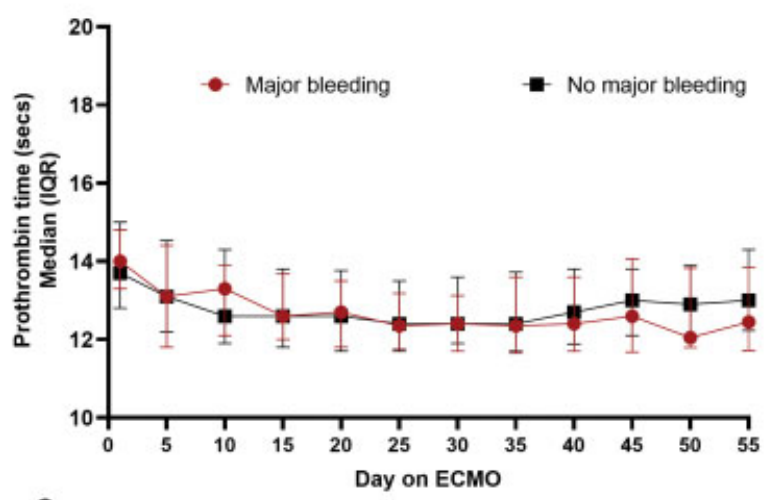

C

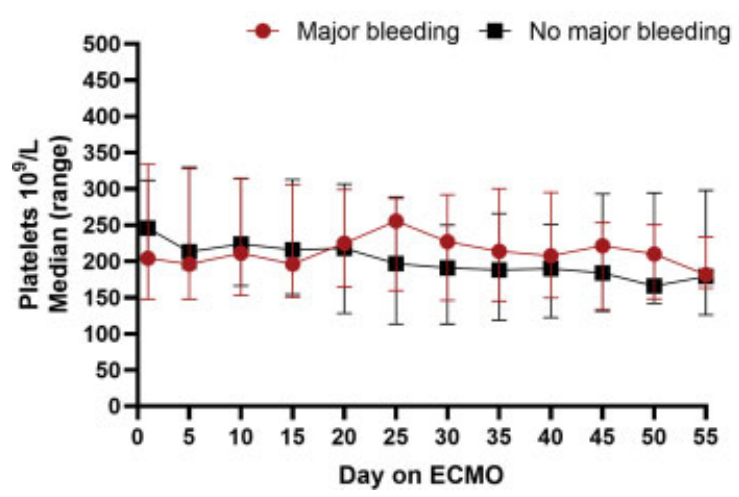

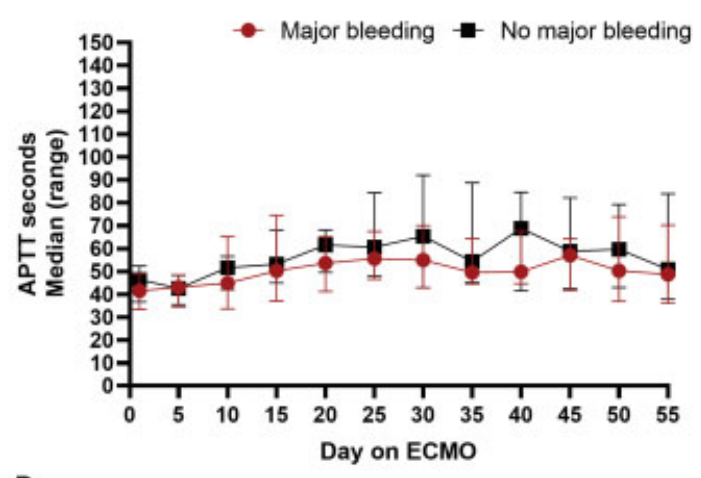

D

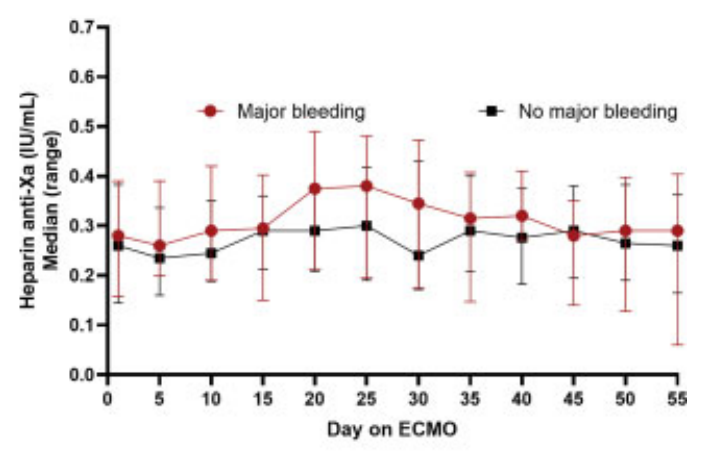

Fig. 1 (A-D) Prothrombin time, aPTT, platelet count, and heparin anti-Xa level over time on ECMO in patients with COVID-19 pneumonia who developed major bleeding versus no major bleeding. aPTT, activated partial thromboplastin time; COVID-19, coronavirus disease 2019; ECMO, extracorporeal membrane oxygenation.

supported with VV-ECMO. Our principal finding is that although thrombosis is a dominant feature of COVID-19 infection, in patients supported with VV-ECMO, bleeding was the major cause of death, accounting for five out of nine deaths in COVID-19, compared to none in non-COVID-19 patients. Nonetheless, thrombosis was identified in $80 \%$ of the COVID19 cohort compared to $60 \%$ in the non-COVID-19 group, supporting a large body of evidence that thrombosis, particularly in the pulmonary circulation, is a distinctly prominent complication of severe COVID-19., ${ }^{7,8}$ The additional thrombotic risk conferred by COVID-19 was apparent not only before, but also during VV-ECMO, despite standardized thromboprophylaxis. This is supported by the report of our neighboring ECMO center which compared 51 patients supported with ECMO for COVID-19 with 80 patients with influenza. ${ }^{9}$ The longer duration of VV-ECMO in the COVID-19 cohort may have biased results, but the majority (60/69) of thrombotic events occurred in $<15$ days of VV-ECMO-the median duration of VV-ECMO in the non-COVID-19 cohort. The significantly higher neutrophil count and fibrinogen levels may have contributed to the higher rates of thrombosis.

Major and minor hemorrhages, particularly ICH, were numerically more prevalent in the COVID-19 cohort, despite higher platelet count and fibrinogen levels on admission. Major bleeding secondary to thrombosis was the cause of death in five of nine COVID-19 patients who died (-Supplementary Table S3, available in the online version only), while bleeding was not associated with any deaths in the non-COVID-19 cohort ( - Supplementary Table S4, available in the online version only). Usman et al published a small report including 10 patients receiving VV-ECMO for ARDS secondary to COVID-19 and four of their 10 patients developed ICH, three of which were fatal. ${ }^{10}$

Although ICH is a recognized general complication of VVECMO and is associated with early mortality ${ }^{3}$ the explanation for the increased ICH in COVID-19 is not clear. ${ }^{3,11}$ Thrombocytopenia and renal failure were previously identified as risk factors but were not more prominent in our COVID-19 cohort. By contrast, the patients with COVID-19 had higher platelet count and fibrinogen levels at the admission and platelet count was persistently above $100 \times 10^{9} / \mathrm{L}$ throughout the ECMO support ( $\mathbf{- F i g . ~ 1 C ) . ~ A ~ p o s s i b l e ~ e x p l a - ~}$ nation is the greater thrombotic burden necessitating therapeutic anticoagulation in more patients. Indeed, in the COVID-19 cohort, 16/23 major hemorrhages occurred in the context of treatment-dose anticoagulation.

Despite more thrombotic and hemorrhagic complications in the COVID-19 cohort, there was no significant difference in overall mortality between the two cohorts at 120 days. The lower prevalence of RRT and multiorgan failure in the COVID-19 cohort may help explain this. The majority of patients in the COVID-19 cohort died due to ICH and death occurred relatively earlier during ECMO compared to non- 
A

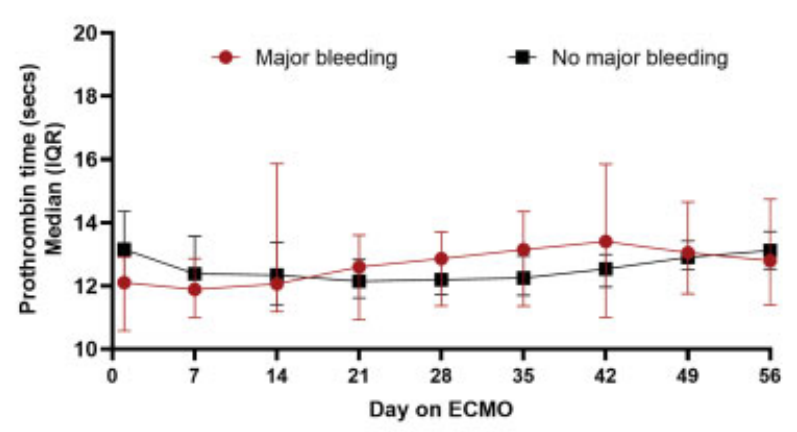

C

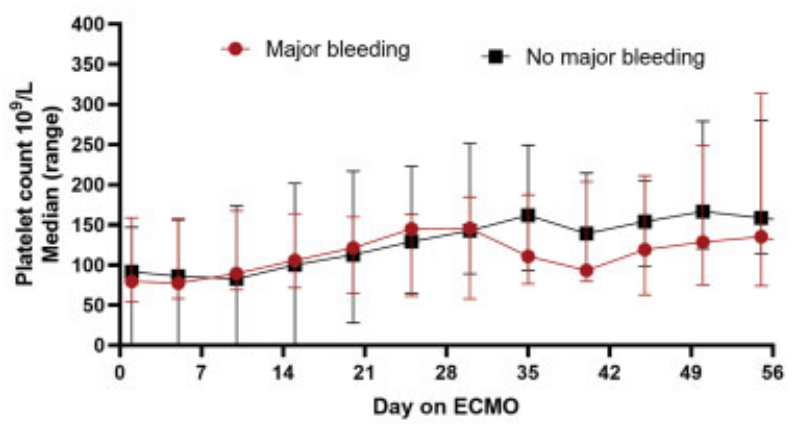

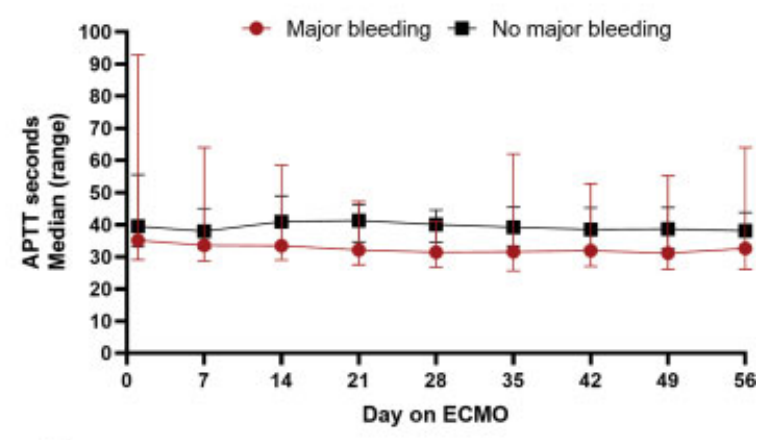

D

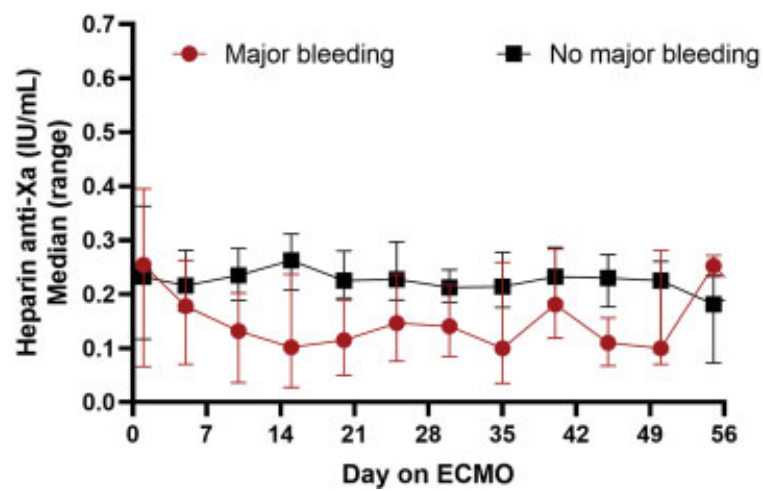

Fig. 2 (A-D) Prothrombin time, aPTT, platelet count, and heparin anti-Xa level over time on ECMO in patients with non-COVID-19 viral pneumonia who developed major bleeding versus no major bleeding. aPTT, activated partial thromboplastin time; COVID-19, coronavirus disease 2019; ECMO, extracorporeal membrane oxygenation.

COVID-19 patients (-Supplementary Tables $\mathbf{5 3}$ and S4, available in the online version only).

This study's main limitations are its retrospective nature and small sample sizes. However, it does give a detailed description of thrombosis and bleeding in patients receiving VV-ECMO for COVID-19 ARDS compared to a comparable historical cohort receiving VV-ECMO for non-COVID-19 viral pneumonia, with standardized imaging and anticoagulation protocols.

We extend the body of evidence that the risks of thrombosis and hemorrhage are higher in patients with COVID-19 compared to non-COVID-19 viral pneumonia supported with VV-ECMO. The pattern of timing of thrombotic events predominantly prior to and early in the course of VV-ECMO in the COVID-19 cohort supports the hypothesis that active COVID-19 was a key driver of thrombosis in this group. Major hemorrhage was an important cause of death in the COVID19 cohort alone. Vigilant monitoring for major bleeding is required in patients with COVID-19 supported with VVECMO, at least in part due to the high doses of anticoagulation required to treat often recurrent thrombosis.

\section{Authors' Contributions}

A.W. was involved in data collection, data interpretation, writing the original draft, and editing the manuscript. D.J. A. designed the study, involved in data collection, data verification, data analysis, data interpretation, figures and writing the original draft reviewing and editing the manuscript. M.L. contributed by interpretation of the data and editing the manuscript. M.G. contributed to data collection and reviewing the manuscript. B.G. contributed by data verification and revising the manuscript. Others contributed by interpretation of the data and editing the manuscript. All authors critically reviewed and approved the final manuscript.

Conflict of Interest

None declared.

Acknowledgments

The authors would like to thank all clinical staff at adult intensive care units, transfusion and the laboratory staff at Royal Brompton and Harefield hospitals.

\section{References}

1 Barbaro RP, MacLaren G, Boonstra PS, et al; Extracorporeal Life Support Organization. Extracorporeal membrane oxygenation support in COVID-19: an international cohort study of the Extracorporeal Life Support Organization registry. Lancet 2020;396 (10257):1071-1078

2 Olson SR, Murphree CR, Zonies D, et al. Thrombosis and bleeding in extracorporeal membrane oxygenation (ECMO) without anticoagulation: a systematic review. ASAIO J 2021;67(03):290-296

3 Arachchillage DRJ, Passariello M, Laffan $M$, et al. Intracranial hemorrhage and early mortality in patients receiving extracorporeal membrane oxygenation for severe respiratory failure. Semin Thromb Hemost 2018;44(03):276-286 
4 Arachchillage DJ, Remmington C, Rosenberg A, et al. Anticoagulation with argatroban in patients with acute antithrombin deficiency in severe COVID-19. Br J Haematol 2020;190(05):e286-e288

5 Schulman S, Kearon CSubcommittee on Control of Anticoagulation of the Scientific and Standardization Committee of the International Society on Thrombosis and Haemostasis. Definition of major bleeding in clinical investigations of antihemostatic medicinal products in non-surgical patients. J Thromb Haemost 2005;3(04):692-694

6 Kaatz S, Ahmad D, Spyropoulos AC, Schulman SSubcommittee on Control of Anticoagulation. Definition of clinically relevant nonmajor bleeding in studies of anticoagulants in atrial fibrillation and venous thromboembolic disease in non-surgical patients: communication from the SSC of the ISTH. J Thromb Haemost 2015;13(11):2119-2126

7 Helms J, Tacquard C, Severac F, et al; CRICS TRIGGERSEP Group (Clinical Research in Intensive Care and Sepsis Trial Group for Global Evaluation and Research in Sepsis) High risk of thrombosis in patients with severe SARS-CoV-2 infection: a multicenter prospective cohort study. Intensive Care Med 2020;46(06): 1089-1098

8 Taccone FS, Gevenois PA, Peluso L, et al. Higher intensity thromboprophylaxis regimens and pulmonary embolism in critically ill coronavirus disease 2019 patients. Crit Care Med 2020;48(11): e1087-e1090

9 Doyle AJ, Hunt BJ, Sanderson B, et al. A comparison of thrombosis and hemorrhage rates in patients with severe respiratory failure due to coronavirus disease 2019 and influenza requiring extracorporeal membrane oxygenation. Crit Care Med 2021;49(07): e663-e672

10 Usman AA, Han J, Acker A, et al. A case series of devastating intracranial hemorrhage during venovenous extracorporeal membrane oxygenation for COVID-19. J Cardiothorac Vasc Anesth 2020;34(11):3006-3012

11 Luyt $\mathrm{CE}$, Bréchot $\mathrm{N}$, Demondion $\mathrm{P}$, et al. Brain injury during venovenous extracorporeal membrane oxygenation. Intensive Care Med 2016;42(05):897-907 